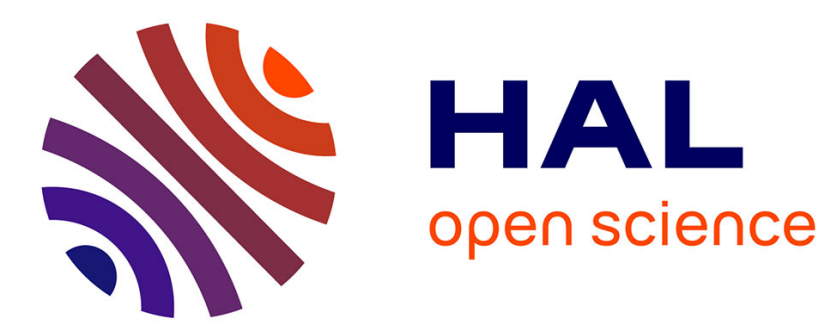

\title{
Curvature induced magnetic bound states: towards the tunneling effect for the ellipse
}

Virginie Bonnaillie-Noël, Frédéric Hérau, Nicolas Raymond

\section{To cite this version:}

Virginie Bonnaillie-Noël, Frédéric Hérau, Nicolas Raymond. Curvature induced magnetic bound states: towards the tunneling effect for the ellipse. 2017. hal-01404924v2

\section{HAL Id: hal-01404924 \\ https://hal.science/hal-01404924v2}

Preprint submitted on 20 Jan 2017

HAL is a multi-disciplinary open access archive for the deposit and dissemination of scientific research documents, whether they are published or not. The documents may come from teaching and research institutions in France or abroad, or from public or private research centers.
L'archive ouverte pluridisciplinaire HAL, est destinée au dépôt et à la diffusion de documents scientifiques de niveau recherche, publiés ou non, émanant des établissements d'enseignement et de recherche français ou étrangers, des laboratoires publics ou privés. 


\title{
Curvature induced magnetic bound states: towards the tunneling effect for the ellipse
}

\author{
VIRGINIE BONNAILLIE-NOËL \\ FrÉDÉRIC HÉRAU \\ NiCOLAS RAYMOND
}

\begin{abstract}
This article is devoted to the semiclassical analysis of the magnetic Laplacian on a smooth domain of the plane carrying Neumann boundary conditions. We provide WKB expansions of the eigenfunctions when Neumann boundary traps the lowest eigenfunctions near the points of maximal curvature. We also explain and illustrate a conjecture of magnetic tunneling when the domain is an ellipse.
\end{abstract}

\section{Introduction}

Let $\Omega$ be an open, bounded and simply connected domain of $\mathbb{R}^{2}$. We consider the magnetic Laplacian

$$
\mathcal{L}_{\hbar}=(-i \hbar \nabla+\mathbf{A})^{2},
$$

with Neumann condition on the boundary, where $\mathbf{A}\left(x_{1}, x_{2}\right)=\frac{1}{2}\left(x_{2},-x_{1}\right)$ is a vector potential associated with the constant magnetic field $\mathbf{B}=\nabla \times \mathbf{A}=1$. Here $\hbar$ is a small positive parameter. In the following, we will mainly consider the case when $\Omega$ is smooth and we denote $|\partial \Omega|=\ell$ its perimeter. The operator $\mathcal{L}_{\hbar}$ is the self-adjoint operator associated with the closed quadratic form defined for $\psi \in \mathrm{H}^{1}(\Omega)$ by

$$
Q_{\hbar}(\psi)=\int_{\Omega}|(-i \hbar \nabla+\mathbf{A}) \psi|^{2} \mathrm{~d} x_{1} \mathrm{~d} x_{2} .
$$

The aim of this article is to present some recent results about the low lying eigenvalues of $\mathcal{L}_{\hbar}$ and their associated (quasi)modes, especially when there are symmetries and multiple points of maximal curvature on the boundary, and we will focus on the case of ellipses.

\subsection{Semiclassical spectral gap}

We are interested in the non-decreasing sequence of the eigenvalues of $\mathcal{L}_{\hbar}$, denoted by $\lambda_{n}(\hbar)$, and especially in the gap between the first two ones $\lambda_{2}(\hbar)-\lambda_{1}(\hbar)$. The question of estimating the gap between the magnetic eigenvalues was initially raised in [8] in the case of constant magnetic fields in two dimensions. Fournais and Helffer have shown the fundamental role of the curvature of the boundary in the semiclassical expansion of the gap (and improved the previous contribution by Helffer and Morame [13] where only the first eigenvalue was considered). Let us recall their result.

Theorem 1.1. Let us assume that the algebraic curvature $\kappa$ of the boundary of $\partial \Omega$ has a unique and non-degenerate maximum (attained at a point of the boundary with curvilinear abscissa 0 ). Then, we have the asymptotic expansion

$$
\lambda_{n}(\hbar)=\Theta_{0} \hbar-C_{1} \kappa_{\max } \hbar^{3 / 2}+(2 n-1) C_{1} \Theta_{0}^{1 / 4} \sqrt{\frac{3 k_{2}}{2}} \hbar^{7 / 4}+o\left(\hbar^{7 / 4}\right),
$$

where $\Theta_{0} \in(0,1)$ and $C_{1}>0$ are constants related to the De Gennes operator (see Proposition 3.2) and $k_{2}=-\kappa^{\prime \prime}(0)$. 


\section{Bonnaillie-NoëL, F. HÉrau, \& N. Raymond}

In $[17,7]$, the authors have estimated the spectral gap in the case of varying magnetic fields in two dimensions. They have shown that the spectral asymptotics was governed by an effective magnetic curvature and that a dimensional reduction in the spirit of the famous Born-Oppenheimer approximation was a key to determine the spectral gap. Note that this gap was also estimated in [11] (see also [12]) via a Grushin-like reduction or in [19] via a semiclassical Birkhoff normal form. We refer to the books [9] and [18] where extensive bibliographic references can be found about this question.

\subsection{WKB expansions}

The problematics of estimating the semiclassical spectral gap is closely related to WKB constructions. These constructions were already known for the Schrödinger operator with electric potential (see for instance [14, 6]). Nevertheless, until now, not even an example of such constructions was provided in the case of pure magnetic fields. This was the aim of our contribution [3] to show, in a generic and multi-scale framework, that the magnetic eigenfunctions have (complex) WKB expansions. In [3], we developed a theory in the Born-Oppenheimer spirit to estimate the spectral gap and ensure the existence of WKB constructions. We will not reproduce in the present paper this general framework, but only discuss it in the same context as Theorem 1.1. We will explain formally why the following operator, acting on $\mathrm{L}^{2}(\mathbb{R} / \ell \mathbb{Z}, \mathrm{d} s)$, determines the semiclassical spectral asymptotics:

$$
\mathcal{L}_{\hbar}^{\text {eff }}=\Theta_{0} \hbar+\frac{\mu^{\prime \prime}\left(\zeta_{0}\right)}{2}\left(\hbar D_{s}+\gamma_{0}-\zeta_{0} \hbar^{\frac{1}{2}}+\alpha_{0} \hbar\right)^{2}-C_{1} \kappa(s) \hbar^{\frac{3}{2}}, \quad \gamma_{0}=\frac{|\Omega|}{\ell},
$$

where $D_{s}=-i \partial_{s}, \ell=|\partial \Omega|, \zeta_{0}=\sqrt{\Theta_{0}}$ and $\alpha_{0}$ is a constant related to the De Gennes operator (see Proposition 3.2 for some properties of the De Gennes operator).

Remark 1.2. The spectral behavior of $\mathcal{L}_{\hbar}^{\text {eff }}$ is well known. If $\kappa$ has a unique and nondegenerate maximum, then the first eigenfunctions are localized near this maximum and a local change of gauge reduces the investigation to

$$
\Theta_{0} \hbar+\frac{\mu^{\prime \prime}\left(\zeta_{0}\right)}{2} \hbar^{2} D_{s}^{2}-C_{1} \kappa(s) \hbar^{\frac{3}{2}}
$$

for which the usual harmonic approximation applies (as well as the WKB constructions). If $\kappa$ has two symmetric maxima, such a change of gauge is not allowed since there is, in general, no global change of gauge to cancel the flux term (there is a phase shift between the two wells, see [16] and [4]). The symmetry of $\kappa$ reveals then a global phenomenon.

The following result is a local WKB construction (near the unique maximum of the curvature) reflecting the formal approximation by the effective operator (1.4). In the statement we will use the classical tubular coordinates $(s, t)$ near the boundary (see the precise derivation in Section 2 below).

Theorem 1.3 (WKB form, Curvature induced magnetic bound states). There exist an explicit function $\Phi=\Phi(s)$ defined in a neighborhood $\mathcal{V}$ of $(0,0)$ such that $\operatorname{Re} \Phi^{\prime \prime}(0)>0$, and a sequence of real numbers $\left(\lambda_{n, j}\right)_{j \geq 0}$ such that

$$
\lambda_{n}(\hbar) \underset{\hbar \rightarrow 0}{\sim} \hbar \sum_{j \geq 0} \lambda_{n, j} \hbar^{\frac{j}{4}}
$$

with $\lambda_{n, 0}=\Theta_{0}, \lambda_{n, 1}=0, \lambda_{n, 2}=-C_{1} \kappa_{\max }$ and $\lambda_{n, 3}=(2 n-1) C_{1} \Theta_{0}^{1 / 4} \sqrt{\frac{3 k_{2}}{2}}$. Besides there exists a formal series of smooth functions $a_{n} \underset{\hbar \rightarrow 0}{\sim} \sum_{j \geq 0} a_{n, j} \hbar^{\frac{j}{4}}$ on $\mathcal{V}$ such that

$$
\left(\mathcal{L}_{\hbar}-\lambda_{n}(\hbar)\right)\left(\mathrm{a}_{n} \mathrm{e}^{-\Phi / \hbar^{\frac{1}{4}}}\right)=\mathcal{O}\left(\hbar^{\infty}\right) \mathrm{e}^{-\Phi / \hbar^{\frac{1}{4}}} .
$$




\section{CURVATURE INDUCED MAGNETIC BOUND STATES}

The main term in the Ansatz is in the tensorial form $\mathrm{a}_{n, 0}(s, t)=f_{n, 0}(s) u_{\zeta_{0}}\left(\hbar^{-\frac{1}{2}} t\right)$. Moreover, for all $n \geq 1$, there exist $\hbar_{0}>0$ and $c>0$ such that for all $\hbar \in\left(0, \hbar_{0}\right)$, we have

$$
\mathcal{B}\left(\Theta_{0} \hbar-C_{1} \kappa_{\max } \hbar^{\frac{3}{2}}+\lambda_{n, 3} \hbar^{\frac{7}{4}}, c \hbar^{\frac{7}{4}}\right) \cap \operatorname{sp}\left(\mathcal{L}_{\hbar}\right)=\left\{\lambda_{n}(\hbar)\right\}
$$

and $\lambda_{n}(\hbar)$ is a simple eigenvalue. Here $\mathcal{B}(a, \rho)$ denotes the ball centered at a of radius $\rho$.

\subsection{Tunneling effect for the ellipse}

Considering the effective operator (1.3) and Remark 1.2 lead to the following conjecture in the case when $\Omega$ is an ellipse. We consider

$$
\Omega=\left\{\left(x_{1}, x_{2}\right) \in \mathbb{R}^{2}: \frac{x_{1}^{2}}{a^{2}}+\frac{x_{2}^{2}}{b^{2}}<1\right\}, \quad a>b>0,
$$

recall that $\ell=|\partial \Omega|, k_{2}=-\kappa^{\prime \prime}(0)$ and assume that the curvilinear abscissa of $(a, 0)$ is 0 .

Conjecture 1.4.

$\lambda_{2}(\hbar)-\lambda_{1}(\hbar) \underset{\hbar \rightarrow 0}{\sim} \hbar^{\frac{13}{8}} \frac{2^{\frac{5}{2}}}{\sqrt{\pi}}\left(k_{2} \mu^{\prime \prime}\left(\zeta_{0}\right)\right)^{\frac{1}{4}}\left(\kappa(0)-\kappa\left(\frac{\ell}{4}\right)\right)^{\frac{1}{2}} C_{1}^{\frac{3}{4}} \mathrm{~A}\left|\cos \left(\frac{\ell}{2}\left(\frac{\gamma_{0}}{\hbar}-\frac{\zeta_{0}}{\hbar^{\frac{1}{2}}}+\alpha_{0}\right)\right)\right| \mathrm{e}^{-\mathrm{S} / \hbar^{\frac{1}{4}}}$,

where

$$
\begin{aligned}
\mathrm{S} & =\sqrt{\frac{2 C_{1}}{\mu^{\prime \prime}\left(\zeta_{0}\right)}} \int_{0}^{\frac{\ell}{2}} \sqrt{\kappa(0)-\kappa(s)} \mathrm{d} s, \\
\mathrm{~A} & =\exp \left(-\int_{\left[0, \frac{\ell}{4}\right]} \frac{\partial_{s} \sqrt{\kappa(0)-\kappa(s)}-\sqrt{\frac{k_{2}}{2}}}{\sqrt{\kappa(0)-\kappa(s)}} \mathrm{d} s\right) .
\end{aligned}
$$

\subsection{Organization of the paper}

The paper is organized as follows. Section 2 is devoted to the change of coordinates and the corresponding change of operator defined in a tubular neighborhood of the boundary. In Section 3 we recall some well-known facts about the De Gennes operator and apply the result to build an effective operator according to the Born Oppenheimer strategy. In Section 4, we state the main steps of the proof of Theorem 1.3. In Section 5, we recall known results about Schrödinger operators on the circle and apply it to our effective operator, leading to a conjecture about the tunnelling effect for the ellipse. In Section 6, we present numerical simulations illustrating the conjecture.

\section{A global change of coordinates near the boundary}

In this short section we recall the reduction of operator $\mathcal{L}_{\hbar}$ to a multiscale operator defined in a neighborhood of the boundary of $\Omega$. We first write the magnetic Laplacian using tubular coordinates near the boundary.

For $t_{0}>0$ sufficiently small but fixed, let us consider the following smooth neighborhood of $\partial \Omega$ :

$$
\Omega_{t_{0}}=\left\{x \in \Omega, \operatorname{dist}(x, \partial \Omega)<t_{0}\right\},
$$

and $\mathcal{L}_{\hbar}^{\left(t_{0}\right)}$ the realization of $(-i \hbar \nabla+\mathbf{A})^{2}$ with Neumann condition on $\partial \Omega$ and Dirichlet condition on $\left\{x \in \Omega\right.$, dist $\left.(x, \partial \Omega)=t_{0}\right\}$. Using [9, Appendix F], we can rewrite this operator using boundary coordinates. Let $\gamma: \mathbb{R} / \ell \mathbb{Z} \rightarrow \partial \Omega$ be a parametrization of the boundary with $\left|\gamma^{\prime}\right|=1$, and for any $s, \nu(s)$ be the inward unit vector at $\gamma(s)$. The curvature is denoted by $\kappa(s)$ and satisfies

$$
\gamma^{\prime \prime}(s)=\kappa(s) \nu(s) .
$$




\section{Bonnaillie-NoëL, F. HÉrau, \& N. Raymond}

Let us consider the diffeomorphism

$$
\begin{aligned}
\mathrm{F}: \mathbb{R} / \ell \mathbb{Z} & \rightarrow \Omega_{t_{0}} \\
(s, t) & \mapsto \gamma(s)+t \nu(s) .
\end{aligned}
$$

In these new coordinates, the magnetic potential is

$$
\widetilde{\mathbf{A}}(s, t)=\left(\begin{array}{c}
(1-t \kappa(s)) \mathbf{A}(\mathrm{F}(s, t)) \cdot \gamma^{\prime}(s) \\
\mathbf{A}(\mathrm{F}(s, t)) \cdot \nu(s)
\end{array}\right) .
$$

From [9, Lemma F.1.1], there exists a gauge function $\varphi$ (corresponding to a unitary transformation) such that the magnetic potential reads

$$
\widehat{\mathbf{A}}(s, t)=\widetilde{\mathbf{A}}(s, t)-\nabla \varphi(s, t)=\left(\begin{array}{c}
\gamma_{0}-t+\frac{t^{2}}{2} \kappa(s) \\
0
\end{array}\right),
$$

with

$$
\gamma_{0}=\frac{1}{\ell} \int_{\Omega} \operatorname{curl} \mathbf{A}\left(x_{1}, x_{2}\right) \mathrm{d} x_{1} \mathrm{~d} x_{2} .
$$

We have then (see [9, Lemma F.1.1])

Lemma 2.1. The operator $\mathcal{L}_{\hbar}^{\left(t_{0}\right)}$ is unitarily equivalent to the following operator $\widetilde{\mathcal{L}}_{\hbar}^{\left(t_{0}\right)}$ acting on $\mathrm{L}^{2}\left(\mathbb{R} / \ell \mathbb{Z} \times\left(0, t_{0}\right), m(s, t) \mathrm{d} s \mathrm{~d} t\right)$ with Neumann condition on the outer boundary $\{t=0\}$ and Dirichlet condition on the inner boundary $\left\{t=t_{0}\right\}$ :

$$
\begin{aligned}
\widetilde{\mathcal{L}}_{\hbar}^{\left(t_{0}\right)}= & m(s, t)^{-1} \hbar D_{t} m(s, t) \hbar D_{t} \\
& +m(s, t)^{-1}\left(\hbar D_{s}+\gamma_{0}-t+\frac{t^{2}}{2} \kappa(s)\right) m(s, t)^{-1}\left(\hbar D_{s}+\gamma_{0}-t+\frac{t^{2}}{2} \kappa(s)\right),
\end{aligned}
$$

where $D_{s}=-i \partial_{s}$ and $D_{t}=-i \partial_{t}$ and $m(s, t)=1-t \kappa(s)$.

Remark 2.2. Notice that we consider Dirichlet conditions on $\left\{t=t_{0}\right\}$ and the eigenfunctions of $\widetilde{\mathcal{L}}_{\hbar}^{\left(t_{0}\right)}$ are exponentially localized near the boundary $\{t=0\}$ (see e.g. [9]). Then, in some sense, theses eigenfunctions do not see the inner boundary $\left\{t=t_{0}\right\}$.

In the last step, not completely disconnected with this remark, we perform a last change of variables and also consider a new small parameter

$$
h=\hbar^{\frac{1}{2}}, \quad(s, t)=(\sigma, h \tau) .
$$

With this change of variable, we have $\sigma \in \mathbb{R} / \ell \mathbb{Z}$ and $\tau \in I_{h}=\left(0, \frac{t_{0}}{h}\right)$. The operator $\widetilde{\mathcal{L}}_{\hbar}^{\left(t_{0}\right)}$ is thus unitarily equivalent to operator $h^{2} \mathfrak{L}_{h}$ where

$$
\begin{aligned}
& \mathfrak{L}_{h}=m(\sigma, h \tau)^{-1} D_{\tau} m(\sigma, h \tau) D_{\tau} \\
& +m(\sigma, h \tau)^{-1}\left(h D_{\sigma}+\frac{\gamma_{0}}{h}-\tau+h \frac{\tau^{2}}{2} \kappa(\sigma)\right) m(\sigma, h \tau)^{-1}\left(h D_{\sigma}+\frac{\gamma_{0}}{h}-\tau+h \frac{\tau^{2}}{2} \kappa(\sigma)\right),
\end{aligned}
$$

and acting on the space $\mathrm{L}^{2}\left(\mathbb{R} / \ell \mathbb{Z} \times I_{h}, m(\sigma, h \tau) \mathrm{d} \sigma \mathrm{d} \tau\right)$ with Neumann conditions on the boundary $\tau=0$, Dirichlet conditions on the boundary $\tau=t_{0} / h$, and where $m(\sigma, h \tau)=$ $1-h \tau \kappa(\sigma)$. With this expression, the multi-scale behavior is clear since only variable $\sigma$ seems to have a semiclassical behavior. In the following we shall focus on this operator using a Born-Oppenheimer strategy. 


\section{CURVATURE INDUCED MAGNETIC BOUND STATES}

\section{Formal analysis of the operator symbol}

In order to understand Theorem 1.3 and Conjecture 1.4, let us formally describe the mechanism responsible for the WKB constructions.

\subsection{De Gennes operator}

Before analyzing the spectral properties of $\mathfrak{L}_{h}$, let us recall fundamental properties of the De Gennes operator. For $\zeta \in \mathbb{R}$, let us introduce the so-called De Gennes differential operator

$$
\mathcal{H}_{\zeta}=D_{\tau}^{2}+(\tau-\zeta)^{2} .
$$

defined on $\mathrm{L}^{2}\left(\mathbb{R}^{+}\right)$with Neumann conditions on the boundary. We denote by $\mu(\zeta)$ the first eigenvalue of this operator and by $u_{\zeta}$ a corresponding positive $\mathrm{L}^{2}$-normalized eigenfunction. The behavior of $\mu$ is well known (see, for example, [5], [9, Chapter 3] and [2] for numerical estimates and illustrations). We gather some of its important properties in the following proposition.

Proposition 3.1. The functions $\zeta \mapsto \mu(\zeta)$ and $\zeta \mapsto u_{\zeta}$ are real analytic with respect to $\zeta$. There exists $\zeta_{0}>0$ such that $\mu$ is decreasing on $\left(-\infty, \zeta_{0}\right)$ and increasing on $\left(\zeta_{0},+\infty\right)$, and we have

$$
\Theta_{0}:=\mu\left(\zeta_{0}\right)=\zeta_{0}^{2}, \quad \mu^{\prime}\left(\zeta_{0}\right)=0, \quad\left|u_{\zeta_{0}}(0)\right|^{2}=\frac{\mu^{\prime \prime}\left(\zeta_{0}\right)}{2 \zeta_{0}} .
$$

This is clear from the definition that

$$
\int_{0}^{\infty}\left(\left|u_{\zeta}^{\prime}(\tau)\right|^{2}+(\tau-\zeta)^{2}\left|u_{\zeta}(\tau)\right|^{2}\right) \mathrm{d} \tau=\mu(\zeta)
$$

In fact, we can compute explicitly each term inside the preceding integral and for the second one we recognize a moment of order two. Let us define momenta of the function $u_{\zeta}$ as follows

$$
M_{k}(\zeta)=\int_{0}^{\infty}(\tau-\zeta)^{k}\left|u_{\zeta}(\tau)\right|^{2} \mathrm{~d} \tau, \quad \forall k \in \mathbb{N} .
$$

These moments are calculated in [1] and their expressions are useful to obtain some Feynman-Hellmann type formulas (see, for example, [9, p. 37], [8, p. 10-p. 62]) given in the next proposition

Proposition 3.2. We have

$$
\begin{aligned}
& \int_{\mathbb{R}_{+}}\left(\zeta_{0}-\tau\right) u_{\zeta_{0}}^{2}(\tau) \mathrm{d} \tau=0 \\
& \int_{\mathbb{R}_{+}}\left(\partial_{\zeta} u\right)_{\zeta_{0}}(\tau) u_{\zeta_{0}}(\tau) \mathrm{d} \tau=0 \\
& 2 \int_{\mathbb{R}_{+}}\left(\zeta_{0}-\tau\right)\left(\partial_{\zeta} u\right)_{\zeta_{0}}(\tau) u_{\zeta_{0}}(\tau) \mathrm{d} \tau=\frac{\mu^{\prime \prime}\left(\zeta_{0}\right)}{2}-1, \\
& \int_{\mathbb{R}_{+}}\left(\partial_{\tau}+2 \tau\left(\zeta_{0}-\tau\right)^{2}+\tau^{2}\left(\zeta_{0}-\tau\right)\right) u_{\zeta_{0}} u_{\zeta_{0}} \mathrm{~d} \tau=-C_{1},
\end{aligned}
$$

where

$$
C_{1}=\frac{u_{\zeta_{0}}^{2}(0)}{3}
$$




\section{Bonnaillie-NoëL, F. HÉrau, \& N. Raymond}

Remark 3.3. Using the real analyticity of eigenfuntions and eigenvalues of the De Gennes operator, we directly get that there exists $C_{2} \in \mathbb{R}$ such that, for all $\zeta \in \mathbb{R}$,

$$
\int_{\mathbb{R}_{+}}\left(\partial_{\tau}+2 \tau(\zeta-\tau)^{2}+\tau^{2}(\zeta-\tau)\right) u_{\zeta} u_{\zeta} \mathrm{d} \tau=-C_{1}+C_{2}\left(\zeta-\zeta_{0}\right)+\mathcal{O}\left(\left(\zeta-\zeta_{0}\right)^{2}\right) .
$$

Mention that using the exact expression of the moments in (3.1) and the induced exact expression of the last integral, it is possible to compute explicitly the constant $C_{2}$ in terms of $u_{\zeta}, \mu(\zeta)$ and their derivatives up to order at most 2 evaluated at $\zeta_{0}$.

\subsection{The operator symbol and its lowest eigenvalue}

The operator $\mathfrak{L}_{h}$ defined in (2.6) can be seen as an operator valued operator. Its semiclassical operator symbol in obtained by replacing $h D_{\sigma}+\frac{\gamma_{0}}{h}$ by $\zeta$ and using symbolic calculus. Up to an $\mathcal{O}\left(h^{2}\right)$ error term (which is nevertheless an unbounded operator), the semiclassical operator symbol is given by the following one dimensional operator in the $\tau$-variable, with parameters $\zeta \in \mathbb{R}, \sigma \in \mathbb{R} / \ell \mathbb{Z}, \tau \in I_{h}$,

$$
\begin{aligned}
\mathcal{H}_{\sigma, \zeta, h} & =-m(\sigma, h \tau)^{-1} \partial_{\tau} m(\sigma, h \tau) \partial_{\tau} \\
& +m(\sigma, h \tau)^{-1}\left(\zeta-\tau+h \frac{\tau^{2}}{2} \kappa(\sigma)\right) m(\sigma, h \tau)^{-1}\left(\zeta-\tau+h \frac{\tau^{2}}{2} \kappa(\sigma)\right)+\mathcal{O}\left(h^{2}\right) .
\end{aligned}
$$

We will study this operator thanks to the De Gennes operator and the Feynman-Hellmann formulas recalled previously. We first compute the asymptotic expansion of operator $\mathcal{H}_{\sigma, \zeta, h}$ as $h \rightarrow 0$. Since $m(\sigma, h \tau)=1-h \tau \kappa(\sigma)$, we have for $\sigma \in \mathbb{R} / \ell \mathbb{Z}, \tau \in I_{h}$,

$$
m(\sigma, h \tau)^{-1}=1+h \tau \kappa(\sigma)+\mathcal{O}\left(h^{2}\right) .
$$

According to Remark 2.2 and since $I_{h} \rightarrow \mathbb{R}_{+}$as $h \rightarrow 0$, we consider from now $(\sigma, \tau) \in$ $\mathbb{R} / \ell \mathbb{Z} \times \mathbb{R}_{+}$and replace everywhere $m(\sigma, h \tau)^{-1}$ by $1-h \tau \kappa(\sigma)+\mathcal{O}\left(h^{2}\right)$, supposed to be defined on $\mathbb{R} / \ell \mathbb{Z} \times \mathbb{R}_{+}$. We therefore get the following expression of $\mathcal{H}_{\sigma, \zeta, h}$ for $\sigma \in \mathbb{R} / \ell \mathbb{Z}$ and $\tau \in \mathbb{R}^{+}$,

$$
\begin{aligned}
\mathcal{H}_{\sigma, \zeta, h}=-\left(1+h \tau \kappa(\sigma)+\mathcal{O}\left(h^{2}\right)\right) \partial_{\tau}(1-h \tau \kappa(\sigma)) \partial_{\tau} & \\
& +\left(1+h \tau \kappa(\sigma)+\mathcal{O}\left(h^{2}\right)\right)^{2}\left(\zeta-\tau+h \frac{\tau^{2}}{2} \kappa(\sigma)\right)^{2}+\mathcal{O}\left(h^{2}\right) .
\end{aligned}
$$

We deduce that

$$
\mathcal{H}_{\sigma, \zeta, h}=\mathcal{H}_{\zeta}+h \kappa(\sigma)\left(\partial_{\tau}+2 \tau(\zeta-\tau)^{2}+\tau^{2}(\zeta-\tau)\right)+\mathcal{O}\left(h^{2}\right) .
$$

We can prove that the lowest eigenvalue of operator $\mathcal{H}_{\sigma, \zeta, h}$ is simple and isolated. We denote this eigenvalue $\nu(\sigma, \zeta, h)$. Let us follow the Born-Oppenheimer strategy and compute for each $\sigma \in \mathbb{R} / \ell \mathbb{Z}$ and $\zeta \in \mathbb{R}$ the integral, as $h \rightarrow 0$,

$$
\begin{aligned}
& \int_{0}^{\infty} \mathcal{H}_{\sigma, \zeta, h} u_{\zeta}(\tau) u_{\zeta}(\tau) \mathrm{d} \tau \\
& =\int_{0}^{\infty} \mathcal{H}_{\zeta} u_{\zeta}(\tau) u_{\zeta}(\tau) \mathrm{d} \tau+h \kappa(\sigma) \int_{0}^{\infty}\left(\partial_{\tau}+2 \tau(\zeta-\tau)^{2}+\tau^{2}(\zeta-\tau)\right) u_{\zeta}(\tau) u_{\zeta}(\tau) \mathrm{d} \tau+\mathcal{O}\left(h^{2}\right) .
\end{aligned}
$$

We study each term of this expression. Using formula (3.6) we first write, as $h \rightarrow 0$ and $\zeta \rightarrow \zeta_{0}$,

$$
h \kappa(\sigma) \int_{0}^{\infty}\left(\partial_{\tau}+2 \tau(\zeta-\tau)^{2}+\tau^{2}(\zeta-\tau)\right) u_{\zeta}(\tau) u_{\zeta}(\tau) \mathrm{d} \tau=-C_{1} h \kappa(\sigma)+C_{2} h \kappa(\sigma)\left(\zeta-\zeta_{0}\right)+\mathcal{O}\left(h\left(\zeta-\zeta_{0}\right)^{2}\right) .
$$




\section{CURVATURE INDUCED MAGNETIC BOUND STATES}

Let us recall that eigenfunctions are localized near point of maximal curvature $\kappa_{\max }$. One of them corresponds to $\sigma=0$ and we focus in a first time on this one. By a Taylor formula for $\kappa$ at $\sigma=0$, we write, as $h \rightarrow 0, \sigma \rightarrow 0$ and $\zeta \rightarrow \zeta_{0}$,

$$
\begin{aligned}
h \kappa(\sigma) \int_{0}^{\infty}\left(\partial_{\tau}+\right. & \left.2 \tau(\zeta-\tau)^{2}+\tau^{2}(\zeta-\tau)\right) u_{\zeta}(\tau) u_{\zeta}(\tau) \mathrm{d} \tau \\
= & -C_{1} h \kappa(\sigma)+C_{2} h \kappa_{\max }\left(\zeta-\zeta_{0}\right)+\mathcal{O}\left(h \sigma^{2}\left(\zeta-\zeta_{0}\right)\right)+\mathcal{O}\left(h\left(\zeta-\zeta_{0}\right)^{2}\right) .
\end{aligned}
$$

From Proposition 3.1, the infimum of $\mu$ is reached for $\zeta=\zeta_{0}$ and $\mu\left(\zeta_{0}\right)=\Theta_{0}$. Using the definition of $\mu(\zeta)$ and its Taylor expansion at $\zeta_{0}$, we get, as $\zeta \rightarrow \zeta_{0}$,

$$
\int_{0}^{\infty} \mathcal{H}_{\zeta} u_{\zeta}(\tau) u_{\zeta}(\tau) \mathrm{d} \tau=\mu(\zeta)=\Theta_{0}+\frac{\mu^{\prime \prime}\left(\zeta_{0}\right)}{2}\left(\zeta-\zeta_{0}\right)^{2}+\mathcal{O}\left(\left(\zeta-\zeta_{0}\right)^{3}\right)
$$

Putting these two expressions in (3.9), we get, as $h \rightarrow 0, \sigma \rightarrow 0$ and $\zeta \rightarrow \zeta_{0}$,

$$
\begin{aligned}
\int_{0}^{\infty} \mathcal{H}_{\sigma, \zeta, h} u_{\zeta}(\tau) u_{\zeta}(\tau) \mathrm{d} \tau= & \Theta_{0}+\frac{\mu^{\prime \prime}\left(\zeta_{0}\right)}{2}\left(\zeta-\zeta_{0}\right)^{2}-C_{1} h \kappa(\sigma)+C_{2} h \kappa_{\max }\left(\zeta-\zeta_{0}\right) \\
& +\mathcal{O}\left(h^{2}\right)+\mathcal{O}\left(h \sigma^{2}\left(\zeta-\zeta_{0}\right)\right)+\mathcal{O}\left(h\left(\zeta-\zeta_{0}\right)^{2}\right)+\mathcal{O}\left(\left(\zeta-\zeta_{0}\right)^{3}\right) \\
= & \Theta_{0}+\frac{\mu^{\prime \prime}\left(\zeta_{0}\right)}{2}\left(\zeta-\zeta_{0}+\alpha_{0} h\right)^{2}-C_{1} h \kappa(\sigma) \\
& +\mathcal{O}\left(h^{2}\right)+\mathcal{O}\left(h \sigma^{2}\left(\zeta-\zeta_{0}\right)\right)+\mathcal{O}\left(h\left(\zeta-\zeta_{0}\right)^{2}\right)+\mathcal{O}\left(\left(\zeta-\zeta_{0}\right)^{3}\right),
\end{aligned}
$$

where $\alpha_{0}$ is defined by $\mu^{\prime \prime}\left(\zeta_{0}\right) \alpha_{0}=C_{2} \kappa_{\max }$.

Let us now define $\mathcal{M}=\left\{\sigma_{1}, \sigma_{2}, \cdots, \sigma_{N}\right\}$ the set of all curvilinear abcissa where the maximal curvature $\kappa_{\max }$ is attained (we can suppose for example that $\sigma_{1}=0$ ). The preceding asymptotics remain true with $\sigma \rightarrow 0$ replaced by $\operatorname{dist}(\sigma, \mathcal{M}) \rightarrow 0$, where $\operatorname{dist}(\sigma, \mathcal{M})$ stands for the curvilinear distance between $\sigma$ and the set $\mathcal{M}$.

Therefore, at a formal level, and coming back to operators in variable $\sigma$, one expects that the low lying spectrum of the operator $\mathfrak{L}_{h}$ should be asymptotically the same as the one of

$$
\mathfrak{L}_{h}^{\mathrm{eff}}=\Theta_{0}+\frac{\mu^{\prime \prime}\left(\zeta_{0}\right)}{2}\left(h D_{\sigma}+\frac{\gamma_{0}}{h}-\zeta_{0}+\alpha_{0} h\right)^{2}-C_{1} \kappa(\sigma) h,
$$

acting on $\mathrm{L}^{2}(\mathbb{R} / \ell \mathbb{Z}, \mathrm{d} \sigma)$, and up to operators with symbol

$$
\mathcal{O}\left(h^{2}\right), \quad \mathcal{O}\left(h(\operatorname{dist}(\sigma, \mathcal{M}))^{2}\left(\zeta-\zeta_{0}\right)\right), \quad \mathcal{O}\left(h\left(\zeta-\zeta_{0}\right)^{2}\right) \quad \text { and } \quad \mathcal{O}\left(\left(\zeta-\zeta_{0}\right)^{3}\right) .
$$

The operator defined in (3.10) appears to be a magnetic Schrödinger operator with a smooth potential on $\mathbb{R} / \ell \mathbb{Z}$. After rescaling, we get the effective operator (1.3).

\section{WKB constructions in the simple well case}

Let us now explain the main steps in the proof of Theorem 1.3. In fact it is a direct consequence of the change of variables (2.5) and of the following result from [3] on the rescaled operator $\mathfrak{L}_{h}$ defined in (2.6). We denote $\lambda_{n}(h)$ the $n$-th eigenvalue of $\mathfrak{L}_{h}$.

Theorem 4.1. There exist a function

$$
\Phi: \sigma \mapsto \Phi(\sigma)=\left(\frac{2 C_{1}}{\mu^{\prime \prime}\left(\zeta_{0}\right)}\right)^{1 / 2}\left|\int_{0}^{\sigma}(\kappa(0)-\kappa(\varsigma))^{1 / 2} \mathrm{~d} \varsigma\right|,
$$

defined in a neighborhood $\mathcal{V}$ of $(0,0)$ such that $\operatorname{Re} \Phi^{\prime \prime}(0)>0$, and a sequence of real numbers $\left(\lambda_{n, j}\right)_{j \geq 0}$ such that

$$
\lambda_{n}(h) \underset{h \rightarrow 0}{\sim} \sum_{j \geq 0} \lambda_{n, j} h^{\frac{j}{2}} .
$$




\section{Bonnaillie-NoëL, F. HÉrau, \& N. Raymond}

Besides there exists a formal series of smooth functions on $\mathcal{V}$,

$$
a_{n} \underset{h \rightarrow 0}{\sim} \sum_{j \geq 0} a_{n, j} h^{\frac{j}{2}}
$$

such that

$$
\left(\mathfrak{L}_{h}-\lambda_{n}(\hbar)\right)\left(a_{n} \mathrm{e}^{-\Phi / h^{\frac{1}{2}}}\right)=\mathcal{O}\left(h^{\infty}\right) \mathrm{e}^{-\Phi / h^{\frac{1}{2}}} .
$$

We also have that $\lambda_{n, 0}=\Theta_{0}, \lambda_{n, 1}=0, \lambda_{n, 2}=-C_{1} \kappa_{\max }$ and $\lambda_{n, 3}=(2 n-1) C_{1} \Theta_{0}^{1 / 4} \sqrt{\frac{3 k_{2}}{2}}$. The main term in the Ansatz is in the form

$$
a_{n, 0}(\sigma, \tau)=f_{n, 0}(\sigma) u_{\zeta_{0}}(\tau)
$$

Moreover, for all $n \geq 1$, there exist $h_{0}>0, c>0$ such that for all $h \in\left(0, h_{0}\right)$, we have

$$
\mathcal{B}\left(\lambda_{n, 0}+\lambda_{n, 2} h+\lambda_{n, 3} h^{\frac{3}{2}}, c h^{\frac{3}{2}}\right) \cap \operatorname{sp}\left(\mathfrak{L}_{h}\right)=\left\{\lambda_{n}(h)\right\},
$$

and $\lambda_{n}(h)$ is a simple eigenvalue.

Sketch of proof. Let us give some elements of proof based on [3]. Let us introduce a phase function $\Phi=\Phi(\sigma)$ defined in a neighborhood of $\sigma=0$ which is the unique and non degenerate maximum of the curvature $\kappa=\kappa(0)$. We consider the conjugate operator

$$
\mathfrak{L}_{h}^{\mathrm{wg}}=\mathrm{e}^{\Phi(\sigma) / h^{\frac{1}{2}}} \mathfrak{L}_{h} \mathrm{e}^{-\Phi(\sigma) / h^{\frac{1}{2}}} .
$$

As usual, we look for

$$
a \sim \sum_{j \geq 0} h^{\frac{j}{2}} a_{j}, \quad \lambda \sim \sum_{j \geq 0} \lambda_{j} h^{\frac{j}{2}},
$$

such that, in the sense of formal series we have

$$
\mathfrak{L}_{h}^{\mathrm{wg}} a \sim \lambda a .
$$

We may write

$$
\mathfrak{L}_{h}^{\text {wg }} \sim \mathfrak{L}_{0}+h^{\frac{1}{2}} \mathfrak{L}_{1}+h \mathfrak{L}_{2}+h^{\frac{3}{2}} \mathfrak{L}_{3}+\ldots,
$$

where

$$
\begin{aligned}
& \mathfrak{L}_{0}=D_{\tau}^{2}+\left(\zeta_{0}-\tau\right)^{2}, \\
& \mathfrak{L}_{1}=2\left(\zeta_{0}-\tau\right) i \Phi^{\prime}(\sigma), \\
& \mathfrak{L}_{2}=\kappa(\sigma) \partial_{\tau}+2\left(D_{\sigma}+\kappa(\sigma) \frac{\tau^{2}}{2}\right)\left(\zeta_{0}-\tau\right)-\Phi^{\prime}(\sigma)^{2}+2 \kappa(\sigma)\left(\zeta_{0}-\tau\right)^{2} \tau, \\
& \mathfrak{L}_{3}=\left(D_{\sigma}+\kappa(\sigma) \frac{\tau^{2}}{2}\right)\left(i \Phi^{\prime}(\sigma)\right)+\left(i \Phi^{\prime}(\sigma)\right)\left(D_{\sigma}+\kappa(\sigma) \frac{\tau^{2}}{2}\right)+4 i \Phi^{\prime}(\sigma) \tau \kappa(\sigma)\left(\zeta_{0}-\tau\right) .
\end{aligned}
$$

Let us now solve the formal system.

- The first equation is

$$
\mathfrak{L}_{0} a_{0}=\lambda_{0} a_{0},
$$

and leads to take

$$
\lambda_{0}=\Theta_{0}, \quad a_{0}(\sigma, \tau)=f_{0}(\sigma) u_{\zeta_{0}}(\tau),
$$

where $f_{0}$ has to be determined.

- The second equation is

$$
\left(\mathfrak{L}_{0}-\lambda_{0}\right) a_{1}=\left(\lambda_{1}-\mathfrak{L}_{1}\right) a_{0}=\left(\lambda_{1}-2\left(\zeta_{0}-\tau\right) i \Phi^{\prime}(\sigma)\right) u_{\zeta_{0}}(\tau) f_{0}(\sigma) .
$$

Due to the Fredholm alternative, we must take $\lambda_{1}=0$ and

$$
a_{1}(\sigma, \tau)=i \Phi^{\prime}(\sigma) f_{0}(\sigma)\left(\partial_{\zeta} u\right)_{\zeta_{0}}(\tau)+f_{1}(\sigma) u_{\zeta_{0}}(\tau)
$$




\section{CURVATURE INDUCED MAGNETIC BOUND STATES}

where $f_{1}$ is to be determined in a next step.

- Then the third equation is

$$
\left(\mathfrak{L}_{0}-\lambda_{0}\right) a_{2}=\left(\lambda_{2}-\mathfrak{L}_{2}\right) a_{0}-\mathfrak{L}_{1} a_{1} .
$$

Let us explicitly write the right hand side. It equals

$$
\begin{aligned}
\lambda_{2} u_{\zeta_{0}} f_{0}+\Phi^{\prime 2}\left(u_{\zeta_{0}}+2\left(\zeta_{0}-\tau\right)\left(\partial_{\zeta} u\right)_{\zeta_{0}}\right) f_{0}-2\left(\zeta_{0}-\tau\right) u_{\zeta_{0}}\left(i \Phi^{\prime} f_{1}-i \partial_{\sigma} f_{0}\right) & \\
& +\kappa(\sigma) f_{0}\left(\partial_{\tau} u_{\zeta_{0}}-2\left(\zeta_{0}-\tau\right)^{2} \tau u_{\zeta_{0}}-\tau^{2}\left(\zeta_{0}-\tau\right) u_{\zeta_{0}}\right) .
\end{aligned}
$$

Therefore the equation becomes

$$
\left(\mathfrak{L}_{0}-\lambda_{0}\right) \tilde{a}_{2}=\lambda_{2} u_{\zeta_{0}} f_{0}+\frac{\mu^{\prime \prime}\left(\zeta_{0}\right)}{2} \Phi^{\prime 2} u_{\zeta_{0}} f_{0}+\kappa f_{0}\left(-\partial_{\tau} u_{\zeta_{0}}-2\left(\zeta_{0}-\tau\right)^{2} \tau u_{\zeta_{0}}-\tau^{2}\left(\zeta_{0}-\tau\right) u_{\zeta_{0}}\right),
$$

where

$$
\tilde{a}_{2}=a_{2}-\left(\partial_{\zeta} u\right)_{\zeta_{0}}\left(i \Phi^{\prime} f_{1}-i \partial_{\sigma} f_{0}\right)+\frac{1}{2}\left(\partial_{\zeta}^{2} u\right)_{\zeta_{0}} \Phi^{\prime 2} f_{0} .
$$

We now use Proposition 3.2 and get the equation

$$
\lambda_{2}+\frac{\mu^{\prime \prime}\left(\zeta_{0}\right)}{2} \Phi^{\prime 2}(\sigma)+C_{1} \kappa(\sigma)=0, \quad \text { with } \quad C_{1}=\frac{u_{\zeta_{0}}^{2}(0)}{3} .
$$

Here we recognize an eikonal equation of a pure electric problem in dimension one whose potential is given by the curvature. Thus we take

$$
\lambda_{2}=-C_{1} \kappa(0)
$$

and

$$
\Phi(\sigma)=\left(\frac{2 C_{1}}{\mu^{\prime \prime}\left(\zeta_{0}\right)}\right)^{1 / 2}\left|\int_{0}^{\sigma}(\kappa(0)-\kappa(\varsigma))^{1 / 2} \mathrm{~d} \varsigma\right|
$$

In particular we have

$$
\Phi^{\prime \prime}(0)=\left(\frac{k_{2} C_{1}}{\mu^{\prime \prime}\left(\zeta_{0}\right)}\right)^{1 / 2}, \quad \text { with } \quad k_{2}=-\kappa^{\prime \prime}(0)>0 .
$$

This leads to take

$$
a_{2}=f_{0} \hat{a}_{2}+\left(\partial_{\zeta} u\right)_{\zeta_{0}}\left(i \Phi^{\prime} f_{1}-i \partial_{\sigma} f_{0}\right)-\frac{1}{2}\left(\partial_{\eta}^{2} u\right)_{\zeta_{0}} \Phi^{\prime 2} f_{0}+f_{2} u_{\zeta_{0}},
$$

where $\hat{a}_{2}$ is the unique solution, orthogonal to $u_{\zeta_{0}}$ for all $\sigma$, of

$$
\left(\mathfrak{L}_{0}-\lambda_{0}\right) \hat{a}_{2}=\lambda_{2} u_{\zeta_{0}}+\frac{\mu^{\prime \prime}\left(\zeta_{0}\right)}{2} \Phi^{\prime 2} u_{\zeta_{0}}+\kappa\left(-\partial_{\tau} u_{\zeta_{0}}-2\left(\zeta_{0}-\tau\right)^{2} \tau u_{\zeta_{0}}-\tau^{2}\left(\zeta_{0}-\tau\right) u_{\zeta_{0}}\right),
$$

and $f_{2}$ has to be determined.

- Finally we must solve the fourth equation given by

$$
\left(\mathfrak{L}_{0}-\lambda_{0}\right) a_{3}=\left(\lambda_{3}-\mathfrak{L}_{3}\right) a_{0}+\left(\lambda_{2}-\mathfrak{L}_{2}\right) a_{1}-\mathfrak{L}_{1} a_{2} .
$$

The Fredholm condition provides the following equation in the variable $\sigma$

$$
\left\langle\mathfrak{L}_{3} a_{0}+\left(\mathfrak{L}_{2}-\lambda_{2}\right) a_{1}+\mathfrak{L}_{1} a_{2}, u_{\zeta_{0}}\right\rangle_{\mathrm{L}^{2}\left(\mathbb{R}_{+}, \mathrm{d} \tau\right)}=\lambda_{3} f_{0} .
$$

Using the previous steps of the construction, it is not very difficult to see that this equation does not involve $f_{1}$ and $f_{2}$ (due to the choice of $\Phi$ and $\lambda_{2}$ and Feynman-Hellmann formulas). Using the same formulas, we may write it in the form

$$
\frac{\mu^{\prime \prime}\left(\zeta_{0}\right)}{2}\left(\Phi^{\prime}(\sigma) \partial_{\sigma}+\partial_{\sigma} \Phi^{\prime}(\sigma)\right) f_{0}+F(\sigma) f_{0}=\lambda_{3} f_{0},
$$




\section{Bonnaillie-NoëL, F. HÉrau, \& N. Raymond}

where $F$ is a smooth function which vanishes at $\sigma=0$. Therefore the linearized equation at $\sigma=0$ is given by

$$
\Phi^{\prime \prime}(0) \frac{\mu^{\prime \prime}\left(\zeta_{0}\right)}{2}\left(\sigma \partial_{\sigma}+\partial_{\sigma} \sigma\right) f_{0}=\lambda_{3} f_{0} .
$$

We recall that

$$
\frac{\mu^{\prime \prime}\left(\zeta_{0}\right)}{2}=3 C_{1} \Theta_{0}^{1 / 2}
$$

so that the linearized equation becomes

$$
C_{1} \Theta_{0}^{1 / 4} \sqrt{\frac{3 k_{2}}{2}}\left(\sigma \partial_{\sigma}+\partial_{\sigma} \sigma\right) f_{0}=\lambda_{3} f_{0} .
$$

We have to choose $\lambda_{3}$ in the spectrum of this transport equation, which is given by the set

$$
\left\{(2 n-1) C_{1} \Theta_{0}^{1 / 4} \sqrt{\frac{3 k_{2}}{2}}, \quad n \geq 1\right\} .
$$

If $\lambda_{3}$ belongs to this set, we may solve locally the transport equation (4.1) and thus find $f_{0}$.

This procedure can be continued at any order.

To deduce Theorem 1.3, it is enough to notice that $\lambda_{n}(\hbar)=h^{2} \lambda_{n}(h)$ and to take $\mathrm{a}_{n}(s, t)=$ $a_{n}(\sigma, \tau)$.

\section{About the conjecture}

Let us finally discuss Conjecture 1.4. As suggested by our formal effective operator (3.10), we first recall a result of tunneling type on a circle from [4].

Let us consider the self-adjoint realization, denoted $\mathfrak{P}_{\varepsilon}$, of the electro-magnetic Laplacian $\left(\varepsilon D_{x}+a(x)\right)^{2}+V(x)$ on $\mathrm{L}_{2 \pi-p e r}^{2}(\mathbb{R}, \mathrm{d} x)$ where the vector potential $a$ and the electric potential $V$ are smooth, $2 \pi$-periodic functions. By a gauge transform, this operator is unitarily equivalent to the following operator

$$
\mathcal{P}_{\varepsilon}=\left(\varepsilon D_{x}+\xi_{0}\right)^{2}+V(x),
$$

with $\xi_{0}=\int_{-\pi}^{\pi} a(x) \mathrm{d} x$.

This type of operator is widely studied in (quasi-)periodic situations, and is one of the stone of the so-called Floquet approach for the corresponding problems. Here we only need to deal with it in the pure periodic case. In [4], we have established an asymptotic result of the splitting between the first two eigenvalues $\rho_{1}(\varepsilon)$ and $\rho_{2}(\varepsilon)$ of $\mathcal{P}_{\varepsilon}$ or $\mathfrak{P}_{\varepsilon}$, when the potential $V$ has two symmetric and non-degenerate wells.

Theorem 5.1. Assume that the function $V$ admits exactly two non-degenerate minima at 0 and $\pi$ with $V(0)=V(\pi)=0$ and satisfies $V(x)=V(\pi-x)=V(-x)$. We let

$$
V_{2}=\sqrt{\frac{V^{\prime \prime}(0)}{2}}
$$

Then, as soon as $\varepsilon$ is small enough, there are only two eigenvalues of $\mathcal{P}_{\varepsilon}$ in the interval $(-\infty, 2 \kappa \varepsilon)$ and they both satisfy

$$
\text { for } j=1,2, \quad \rho_{j}(\varepsilon)=V_{2} \varepsilon+o(\varepsilon) \quad \text { as } \varepsilon \rightarrow 0 .
$$

Let us define the positive Agmon distance and the constant A by

$$
\mathrm{S}=\int_{[0, \pi]} \sqrt{V(x)} \mathrm{d} x, \quad \text { and } \quad \mathrm{A}=\exp \left(-\int_{\left[0, \frac{\pi}{2}\right]} \frac{\partial_{x} \sqrt{V}-V_{2}}{\sqrt{V}} \mathrm{~d} x\right) .
$$




\section{CURVATURE INDUCED MAGNETIC BOUND STATES}

Then we have the spectral gap estimate

$$
\rho_{2}(\varepsilon)-\rho_{1}(\varepsilon)=8 \varepsilon^{1 / 2} \mathrm{~A} \sqrt{V\left(\frac{\pi}{2}\right)} \sqrt{\frac{V_{2}}{\pi}}\left|\cos \left(\frac{\xi_{0} \pi}{\varepsilon}\right)\right| \mathrm{e}^{-\mathrm{S} / \varepsilon}+\varepsilon^{3 / 2} \mathcal{O}\left(\mathrm{e}^{-\mathrm{S} / \varepsilon}\right) .
$$

Note here that this type of result appears in [16] and in [4] where we give its detailed proof in dimension 1 . The main point is that one can see on the previous formula the global topologic effect of the flux $\xi_{0}$.

We now apply the previous result to our effective operator $\mathcal{L}_{\hbar}^{\text {eff }}$ defined in (1.3) which is of the same nature. After a shift by $\Theta_{0} \hbar-C_{1} \kappa(0) \hbar^{\frac{3}{2}}$ and the rescaling $s=\frac{\ell}{2 \pi} x$, the operator $\mathcal{L}_{\hbar}^{\text {eff }}$ becomes the following operator acting on $\mathrm{L}^{2}(\mathbb{R} / 2 \pi \mathbb{Z})$,

$$
\frac{\mu^{\prime \prime}\left(\zeta_{0}\right)}{2} \hbar^{\frac{3}{2}}\left[\left(\varepsilon D_{x}+\xi_{0}\right)^{2}+V(x)\right]
$$

where

$$
\varepsilon=\frac{2 \pi \hbar^{\frac{1}{4}}}{\ell}, \quad \xi_{0}=\frac{\gamma_{0}}{\hbar^{\frac{3}{4}}}-\frac{\zeta_{0}}{\hbar^{\frac{1}{4}}}+\alpha_{0} \hbar^{\frac{1}{4}}, \quad V(x)=\frac{2 C_{1}}{\mu^{\prime \prime}\left(\zeta_{0}\right)}\left(\kappa(0)-\kappa\left(\frac{\ell x}{2 \pi}\right)\right) .
$$

Using Theorem 5.1, we directly get

Proposition 5.2. The spectral gap of the effective operator $\mathcal{L}_{h}^{\text {eff }}$ is given by

$\lambda_{2}^{\text {eff }}(\hbar)-\lambda_{1}^{\text {eff }}(\hbar) \underset{\hbar \rightarrow 0}{\sim} \hbar^{\frac{13}{8}} \frac{2^{\frac{5}{2}}}{\sqrt{\pi}}\left|\kappa^{\prime \prime}(0) \mu^{\prime \prime}\left(\zeta_{0}\right)\right|^{\frac{1}{4}}\left(\kappa(0)-\kappa\left(\frac{\ell}{4}\right)\right)^{\frac{1}{2}} C_{1}^{\frac{3}{4}} \mathrm{~A}\left|\cos \left(\frac{\ell}{2}\left(\frac{\gamma_{0}}{\hbar}-\frac{\zeta_{0}}{\hbar^{\frac{1}{2}}}+\alpha_{0}\right)\right)\right| \mathrm{e}^{-\mathrm{S} / \hbar^{\frac{1}{4}}}$,

where

$$
\begin{aligned}
\mathrm{S} & =\sqrt{\frac{2 C_{1}}{\mu^{\prime \prime}\left(\zeta_{0}\right)}} \int_{0}^{\frac{\ell}{2}} \sqrt{\kappa(0)-\kappa(s)} \mathrm{d} s, \\
\mathrm{~A} & =\exp \left(-\int_{\left[0, \frac{\ell}{4}\right]} \frac{\partial_{s} \sqrt{\kappa(0)-\kappa(s)}-\sqrt{-\frac{\kappa^{\prime \prime}(0)}{2}}}{\sqrt{\kappa(0)-\kappa(s)}} \mathrm{d} s\right) .
\end{aligned}
$$

Conjecture 1.4 says that the spectral gap for the initial problem $\mathcal{L}_{\hbar}$ is the same as the one of the effective operator $\mathcal{L}_{\hbar}^{\text {eff }}$. Of course, many approximations have been done in the previous analysis. In particular, reductions, asymptotic expansions and symbolic computations were done up to $\mathcal{O}\left(h^{2}\right), \mathcal{O}\left(h(\operatorname{dist}(\sigma, \mathcal{M}))^{2}\left(\zeta-\zeta_{0}\right)\right), \mathcal{O}\left(h\left(\zeta-\zeta_{0}\right)^{2}\right)$ or $\mathcal{O}\left(\left(\zeta-\zeta_{0}\right)^{3}\right)$ errors (recall that $\mathcal{M}$ is the set of curvilinear abcissa corresponding to maximal curvature, and note that $\mathcal{M}=\{0, \ell / 2\}$ in the case of the ellipse). This can seem contradictory with the exponentially small effect stated in the conjecture. Such a situation was recently tackled in the context of the Robin Laplacian in [10] where it is proved that, though the effective operator is obtained modulo remainders polynomial in $h$, it governs the tunneling effect thanks to an asymptotic separation of variables. Anyway, as e.g. in the usual study of tunneling in multiple well situations, the eigenvalues themselves may be known with (relatively) rough precision (say $\mathcal{O}\left(h^{N}\right)$ ) whereas the gap is very refined. Perhaps in Conjecture 1.4 some global constants are only approximated since the exact phase of operator $\mathcal{L}_{\hbar}$ is not the same as the (explicit) one of the effective operator. This is the subject of a current work in progress.

\section{Numerical simulations}

The numerical simulations of the spectral gap have been done for the operator $\mathcal{L}_{\hbar}$ defined in (1.1) in the case of the ellipse $\Omega$ defined in (1.5) with $a=2$ and $b=1$. 


\section{Bonnaillie-NoëL, F. HÉrau, \& N. Raymond}

We compute the eigenvalues of $\mathcal{L}_{\hbar}$ with a finite element method (see [15]) with an approximation of degree $\mathbb{Q}_{32}$ on an isoparametric mesh of $\Omega$ with 80 quadrangular elements. Figure 1 illustrates the convergence of the first two eigenvalues and also the expansion (1.2). In these simulations, we take $\hbar \in\{k / 10000,1 \leq k \leq 100\}$. Figure 1 (a) plots $\lambda_{1}(\hbar) / \hbar$ and $\lambda_{2}(\hbar) / \hbar$ according to $1 / \hbar$ and Figure $1(\mathrm{~b})$ represents the difference between the eigenvalues and the main term of the expansion (1.2):

$$
\frac{1}{\hbar} \mapsto \frac{\lambda_{j}(\hbar)}{\hbar}-\Theta_{0}+C_{1} \kappa_{\max } \hbar^{1 / 2}
$$

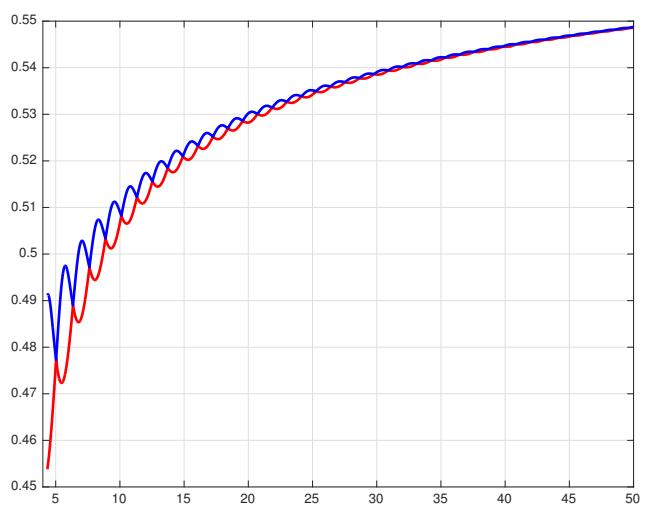

(a) $\lambda_{j}(\hbar) / \hbar$ vs. $1 / \hbar$

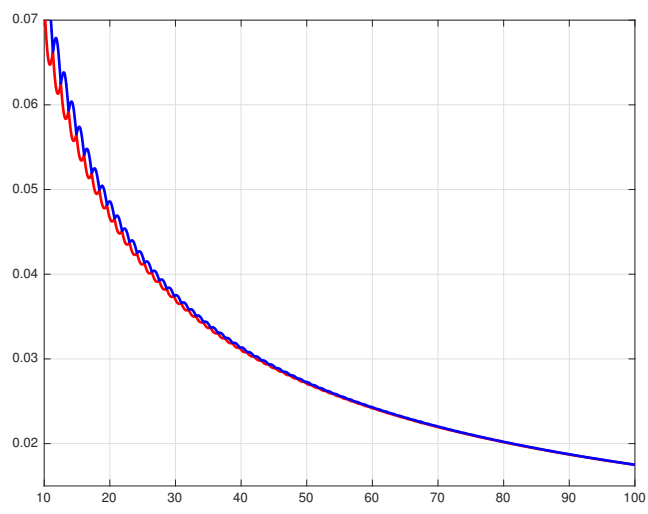

(b) $\lambda_{j}(\hbar) / \hbar-\Theta_{0}+C_{1} \kappa_{\max } \hbar^{1 / 2}$ vs. $1 / \hbar$

Figure 1. Behavior of the first two eigenvalues vs. $1 / \hbar$

Now we will illustrate the tunneling effect and especially Conjecture 1.4 with both the exponential decay and the oscillation between the first two eigenvalues. Thus we have to compute the parameters in Conjecture 1.4. Using the formulas for the ellipse, we have in our situation

$$
\begin{aligned}
|\Omega| & =\pi a b \simeq 6.283, \\
\ell & =4 \int_{0}^{\pi / 2} \sqrt{a^{2} \cos ^{2} t+b^{2} \sin ^{2} t} \mathrm{~d} t \simeq 9.688, \\
e & =\sqrt{1-\frac{b^{2}}{a^{2}}}=\frac{\sqrt{3}}{2},
\end{aligned}
$$

where $e$ denotes the eccentricity. Note that here and in the following, we use a simple composite rectangular rule to compute numerically the integral.

Since we choose a constant magnetic field $\operatorname{curl} \mathbf{A}=1, \gamma_{0}$ defined in (2.3) equals $|\Omega| / \ell$.

The curvilinear parametrization of $\partial \Omega$ and the curvature are then given, for $s \in[0, \ell]$, by

$$
\begin{aligned}
& \gamma(s)=\left(a \cos \left(\frac{2 \pi s}{\ell}\right), b \sin \left(\frac{2 \pi s}{\ell}\right)\right) \\
& \kappa(s)=\frac{b}{a^{2}}\left(1-\mathrm{e}^{2} \cos ^{2}\left(\frac{2 \pi s}{\ell}\right)\right)^{-3 / 2}=\frac{1}{4}\left(1-\frac{3}{4} \cos ^{2}\left(\frac{2 \pi s}{\ell}\right)\right)^{-3 / 2} .
\end{aligned}
$$

The maximum of the curvature is then given by $\kappa_{\max }=\kappa(0)=2$ and $\kappa(\ell / 4)=1 / 4$. We have

$$
\mathrm{S}=\sqrt{\frac{2 C_{1}}{\mu^{\prime \prime}\left(\zeta_{0}\right)}} \frac{\ell}{2 \pi} \frac{\sqrt{b}}{a} \int_{0}^{\pi} \sqrt{\left(1-e^{2}\right)^{-3 / 2}-\left(1-e^{2} \cos ^{2} \mathrm{~s}\right)^{-3 / 2}} \mathrm{~d} \mathrm{~s} \simeq 3.357 .
$$




\section{CURVATURE INDUCED MAGNETIC BOUND STATES}

In Figure 2, we represent the difference between the first two eigenvalues $\lambda_{2}(\hbar)-\lambda_{1}(\hbar)$

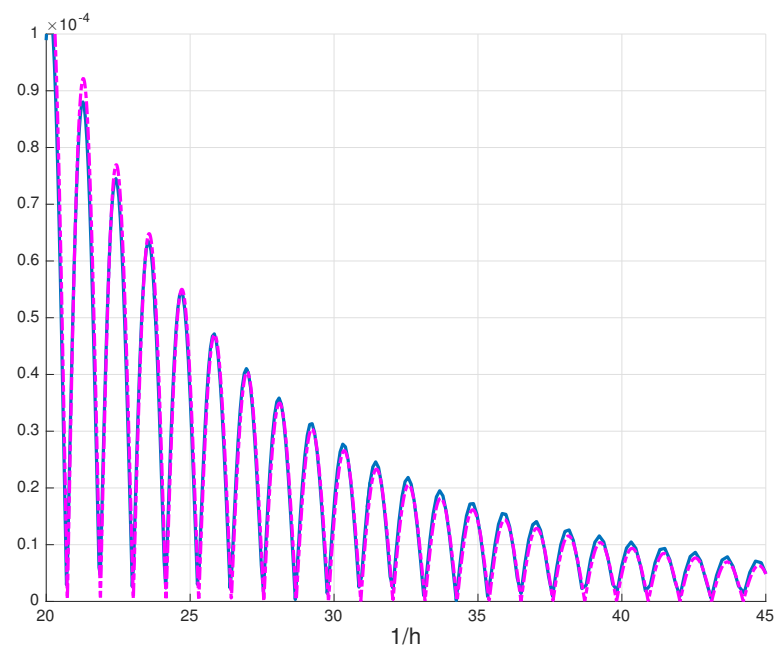

FiguRE 2. Tunneling effect for the ellipse $: \lambda_{2}(\hbar)-\lambda(\hbar)$ and $\delta(\hbar)$ vs. $1 / \hbar$

(in blue) and its conjectured behavior (in pink)

$$
\delta(\hbar)=c \hbar^{\frac{13}{8}}\left|\cos \left(\frac{\ell}{2}\left(\frac{\gamma_{0}}{\hbar}-\frac{\zeta_{0}}{\hbar^{\frac{1}{2}}}+\alpha\right)\right)\right| \mathrm{e}^{-\mathrm{S} / \hbar^{\frac{1}{4}}},
$$

with suitable parameters $c$ and $\alpha$. We observe that our numerical simulations are perfectly coherent with the conjecture.

\section{Bibliography}

[1] A. Bernoff and P. Sternberg. Onset of superconductivity in decreasing fields for general domains. J. Math. Phys., 39(3):1272-1284, 1998.

[2] V. Bonnaillie-Noël. Harmonic oscillators with Neumann condition of the half-line. Commun. Pure Appl. Anal., 11(6):2221-2237, 2012.

[3] V. Bonnaillie-Noël, F. Hérau, and N. Raymond. Magnetic WKB constructions. Arch. Ration. Mech. Anal., 221(2):817-891, 2016.

[4] V. Bonnaillie-Noël, F. Hérau, and N. Raymond. Semiclassical tunneling and magnetic flux effects on the circle. J. Spectr. Theory, page to appear, 2017.

[5] M. Dauge and B. Helffer. Eigenvalues variation. I. Neumann problem for Sturm-Liouville operators. J. Differential Equations, 104(2):243-262, 1993.

[6] M/ Dimassi and J. Sjöstrand. Spectral asymptotics in the semi-classical limit, volume 268 of London Mathematical Society Lecture Note Series. Cambridge University Press, Cambridge, 1999.

[7] N. Dombrowski and N. Raymond. Semiclassical analysis with vanishing magnetic fields. J. Spectr. Theory, 3(3):423-464, 2013.

[8] S. Fournais and B. Helffer. Accurate eigenvalue asymptotics for the magnetic Neumann Laplacian. Ann. Inst. Fourier (Grenoble), 56(1):1-67, 2006.

[9] S. Fournais and B. Helffer. Spectral methods in surface superconductivity. Progress in Nonlinear Differential Equations and their Applications, 77. Birkhäuser Boston Inc., Boston, MA, 2010.

[10] B. Helffer, A. Kachmar, and N. Raymond. Tunneling for the Robin Laplacian in smooth planar domains. To appear in Commun. Contempt. Math. (arXiv:1509.03986), 2016.

[11] B. Helffer and Y. A. Kordyukov. Semiclassical spectral asymptotics for a two-dimensional magnetic Schrödinger operator: the case of discrete wells. In Spectral theory and geometric analysis, volume 535 of Contemp. Math., pages 55-78. Amer. Math. Soc., Providence, RI, 2011.

[12] B. Helffer and Y. A. Kordyukov. Accurate semiclassical spectral asymptotics for a two-dimensional magnetic Schrödinger operator. Ann. Henri Poincaré, 16(7):1651-1688, 2015. 


\section{Bonnaillie-Nö̈L, F. HÉrau, \& N. Raymond}

[13] B. Helffer and A. Morame. Magnetic bottles in connection with superconductivity. J. Funct. Anal., 185(2):604-680, 2001.

[14] B. Helffer and J. Sjöstrand. Multiple wells in the semiclassical limit. I. Comm. Partial Differential Equations, 9(4):337-408, 1984.

[15] D. Martin. Mélina, bibliothèque de calculs éléments finis. http://anum-maths.univ-rennes1.fr/melina, 2010.

[16] A. Outassourt. Comportement semi-classique pour l'opérateur de Schrödinger à potentiel périodique. J. Funct. Anal., 72(1):65-93, 1987.

[17] N. Raymond. From the Laplacian with variable magnetic field to the electric Laplacian in the semiclassical limit. Anal. PDE, 6(6):1289-1326, 2013.

[18] N. Raymond. Bound states of the Magnetic Schrödinger Operator, volume 27 of EMS Tracts in Mathematics. European Mathematical Society, 2017.

[19] N. Raymond and S. Vũ Ngọc. Geometry and spectrum in 2D magnetic wells. Ann. Inst. Fourier (Grenoble), 65(1):137-169, 2015.

VIRGINIE BONNAILLIE-NOËL

DMA - UMR CNRS 8553

PSL Research University

CNRS, ENS Paris

45 rue d'Ulm

F-75230 Paris cedex 05, France

bonnaillie@math.cnrs.fr

NICOLAS RAYMOND

IRMAR - UMR CNRS 8625

Université Rennes 1, CNRS

Campus de Beaulieu

F-35042 Rennes cedex, France

nicolas.raymond@univ-rennes1.fr
FrÉDÉRIC HÉRAU

LMJL - UMR CNRS 6629

Université de Nantes, CNRS

2 rue de la Houssinière

BP 92208

F-44322 Nantes cedex 3, France

frederic.herau@univ-nantes.fr 